\title{
Autoanticorpos na hanseníase: Implicações em diagnóstico e manifestações clínicas. Uma revisão integrativa
}

\author{
Autoantibodies in leprosy: Diagnostic and clinical manifestations. A review \\ Autoanticuerpos en la lepra: Sugerencia en el diagnóstico y manifestaciones clínicas. Una \\ revisión bibliográfica \\ Glaucielen Gomes da Silva1*.
}

\begin{abstract}
RESUMO
Objetivo: Buscar na literatura os autoanticorpos descritos em pacientes com hanseníase nos estudos científicos e sua relação com as manifestações clínicas. Métodos: Trata-se de uma revisão integrativa com busca de artigos indexados na base de dados PubMed em até 10 anos. A busca foi realizada com utilização dos descritores: leprosy, immunomodulation e autoantibodies. Resultados: Foram encontrados 39 artigos, destes, 27 foram excluídos seguindo os critérios de inclusão e exclusão e 12 artigos foram selecionados para responder à questão central desta revisão. Os autoanticorpos citados foram anticorpos anti-fosfolípides (aCL

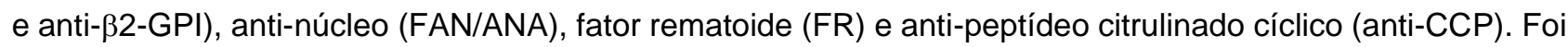
verificado que na maioria dos estudos os pacientes do polo virchowiano apresentaram uma maior positividade para os autoanticorpos. Conclusão: Com base nos resultados podemos verificar que a presença de autoanticorpos, assim como a sintomatologia autoimune, em pacientes com hanseníase pode induzir a um erro de diagnóstico entre esta patologia e doenças reumatológicas. Notou-se ainda a necessidade de estudos que avaliem a relação das manifestações clínicas da hanseníase com os aspectos epidemiológicos e sociais dos pacientes, associando com sintomas autoimunes e positividade para autoanticorpos com o propósito de elucidar características imunomoduladoras do Mycobacterium leprae.
\end{abstract}

.Palavras-Chave: Hanseníase, Imunomodulação, Autoanticorpos.

\begin{abstract}
Objective: To search the literature for autoantibodies and clinical records in leprosy patients in scientific studies and their relationship with clinical manifestations. Methods: This is an review searching for articles indexed in the PubMed database within 10 years. A search was performed using three descriptors: leprosy, immunomodulation and autoantibodies. Results: We found 39 articles, of which 27 were excluded following the inclusion and exclusion criteria and 12 articles were selected to answer the central question of this review. The autoantibodies cited were anti-phospholipids ( $\mathrm{aCL}$ and anti- $\beta 2-\mathrm{GPI}$ ), anti-nucleus (ANA), rheumatoid factor (FR) and anti-cyclic citrullinated peptide (anti-CCP). It has been reported in most studies of lepromatous patients who had a higher positivity for autoantibodies. Conclusion: Based on the results, we can verify that the presence of autoantibodies and autoimmune symptoms, in leprosy patients can induce a misdiagnosis between this pathology and rheumatological diseases. There is still need for studies evaluating a relationship of clinical manifestations of leprosy with the epidemiological and social aspects of patients, associating with autoimmune symptoms and positivity for autoantibodies in order to elucidate immunomodulatory characteristics of Mycobacterium leprae.
\end{abstract}

Key words: Leprosy, Immunomodulation, Autoantibodies.

\footnotetext{
${ }^{1}$ Faculdade dos Carajás, Marabá, Pará. *E-mail: glaucyelen@hotmail.com
} 


\section{RESUMEN}

Objetivo: Buscar en la literatura autoanticuerpos y registros clínicos en pacientes con lepra en estudios científicos y su relación con las manifestaciones clínicas. Métodos: Esta es una revisión de la literatura que busca artículos indexados en la base de datos PubMed dentro de 10 años. La búsqueda se realizó utilizando las palabras clave: lepra, inmunomodulación y autoanticuerpos. Resultados: Encontramos 39 artículos, de los cuales 27 fueron excluidos siguiendo los criterios de inclusión y exclusión y 12 artículos fueron seleccionados para responder la pregunta central de esta revisión. Los autoanticuerpos citados fueron antifosfolípidos (aCL y anti- $\beta 2-G P I)$, antinúcleo (ANA), factor reumatoide (FR) y péptido citrulinado anticíclico (anti-CCP). Se encontró que en la mayoría de los estudios los pacientes con manifestación lepromatosa tenían una mayor positividad para los autoanticuerpos. Conclusión: Con base en los resultados, podemos verificar que la presencia de autoanticuerpos, así como los síntomas autoinmunes, en pacientes con lepra pueden inducir un diagnóstico erróneo entre esta patología y las enfermedades reumatológicas. También se observó la necesidad de estudios que evalúen la relación de las manifestaciones clínicas de la lepra con los aspectos epidemiológicos y sociales de los pacientes, que se asocian con síntomas autoinmunes y positividad para autoanticuerpos con el fin de dilucidar las características inmunomoduladoras de Mycobacterium leprae.

Palabras clave: Lepra, Inmunomodulación, Autoanticuerpos.

\section{INTRODUÇÃO}

A Hanseníase, causada pelo bacilo Mycobacterium leprae, identificado em 1873 por Gerhard Henrik Armauer Hansen, é uma das doenças mais antigas do homem, caracterizada pelo aparecimento de lesões de pele com comprometimento de nervos periféricos. Estima-se que $20 \%$ da população pode desenvolver alterações hansênicas (NAAFS, 2018) que estão envolvidas com a atividade do sistema imunológico do indivíduo (HANSEN GHA, 1875; NAAFS B, 2018)..

Tem sido observado que doenças infecciosas causadas por microrganismos, sejam vírus ou bactérias, podem desencadear uma resposta imunológica contra autoantígenos, originando sintomas de autoimunidade ou até mesmo uma doença autoimune. As doenças autoimunes são desencadeadas quando ocorre perda da autotolerância. Sabemos que fatores genéticos, ambientais e hormonais possuem forte relação na gênese de doenças autoimunes. Entre os mecanismos pelos quais doenças infecciosas geram resposta autoimune, estão o mimetismo molecular, apresentação de antígenos novos, modificados ou crípticos, superantígenos e ativação de linfócitos autorreativos espectadores (HERRATH MG et al., 2003; PERL A, 2004; SAMARKOS M e VAIOPOULOS G, 2005; CHAMBERLIN WM e NASER SA, 2006; SFRISO P et al., 2010; CUSICK MF et al., 2012; QUARESMA JAS et al., 2016; YANG SH et al., 2018).

Diversos estudos apontam que durante o desenvolvimento da hanseníase ocorre a produção e liberação de autoanticorpos que são importantes na apresentação das lesões e reações hansênicas. Durante o processo de ativação da resposta imunológica, envolvendo resposta adaptativa, ocorre a ativação de duas linhas de defesa distintas, atividade adaptativa celular e humoral, desempenhadas por linfócitos $T$ helper 1 (Th1) e T helper 2 (Th2), respectivamente (COSSERMELLI-MESSINA W e COSSERMELLI W, 1995; PERL A, 2004; TALHARI C et al., 2015; SADHU S e MITRA DK, 2018).

As manifestações clínicas da hanseníase foram classificadas e divididas em cinco grupos de acordo com aspectos imunológicos, histológicos e baciloscópicos, sendo duas formas polares; 1 - Tuberculoide (TT), forma neural, paucibacilar, com atividade da resposta adaptativa celular mediada por células T helper 1 (Th1); 2 - Virchowiana (VV), também denominada como Lepromatosa, forma grave, multibacilar, dispersa na pele, com atividade da resposta adaptativa humoral mediada por células $\mathrm{T}$ helper 2 (Th2); A forma clínica intermediária Borderline, também denominada como Dimorfa, é subdividida em três tipos, 3 - BorderlineTuberculoide (BT), semelhante à forma TT se diferenciando no tamanho das lesões e envolvimento neural (TALHARI et al., 2015); 4 - Borderline-Borderline (BB), multibacilar, pode apresentar envolvimento de nervos, 
lesões variadas em tamanhos e formas; 5 - Borderline-Virchowiana (BV), multibacilar com lesões maculares ou nodulares. A forma clínica Indeterminada (I) foi classificada como forma inicial da doença e não se enquadra como um dos grupos de manifestações clínicas da hanseníase (RIDLEY DS e JOPLING WH, 1962, 1966; GUPTA SK, 2015; TALHARI C et al., 2015; RAMAL C, 2017; PAWAR W e ZAWAR V, 2018).

Com base no que foi apresentado, referente à interação do Mycobacterium leprae com a liberação de autoanticorpos, o presente trabalho tem como objetivo realizar um levantamento bibliográfico sobre a presença e relação de autoanticorpos nas manifestações clínicas da hanseníase, respondendo ao questionamento central desta revisão: Quais autoanticorpos já foram descritos sendo sintetizados durante a infecção pelo Mycobacterium leprae?

\section{MÉTODOS}

Este estudo é uma revisão integrativa realizada por meio de levantamento bibliográfico em base de dados científica com o objetivo de responder ao questionamento inicial que é de compreender os mecanismos de autoimunidade na hanseníase através de estudos que relatam o aparecimento de autoanticorpos na infecção causada pelo Mycobacterium leprae.

A coleta de artigos ocorreu no período de 01 a 30 de julho de 2019. Para tanto, foi utilizada como base de dados para pesquisa de artigos científicos o PubMed/MEDLINE da U. S. National Library of Medicine. Os descritores utilizados foram: "hanseníase/leprosy", "antoanticorpos/autoantibodies" e "imunomodulação/immunomodulation", conforme identificados no Descritores de Ciências da Saúde (DeCS) e Medical Subject Headings (MeSH). Os descritores foram combinados entre si para identificação de diversos estudos sobre o referido tema da seguinte forma: ((leprosy) AND autoantibodies) AND immunomodulation.

Foram definidos como critérios de inclusão para esta revisão: utilização de estudos originais, epidemiológicos, observacionais, analíticos e descritivos dos tipos transversal, coorte e estudos de casos clínicos; artigos publicados em inglês ou português; realizados em humanos; indexados na referida base de dados nos últimos 10 anos. Como critérios de exclusão foram definidos: estudos de revisão biliográfica, trabalhos com mais de 10 anos de publicação, e aqueles que não tivessem relação com o questionamento central deste estudo.

\section{RESULTADOS}

Para esta revisão, foram selecionados 12 artigos científicos, baseados nos critérios de inclusão e exclusão descritos anteriormente. Ao aplicar os descritores selecionados, a base de dados PubMed disponibilizou 39 artigos publicados em até 10 anos.

Utilizando os critérios de exclusão a partir da leitura de título e resumo do trabalho, quatro trabalhos foram excluídos por serem revisões bibliográficas e outros 23 trabalhos foram excluídos por não atenderem ao tema da questão principal desta revisão.

Os 12 trabalhos selecionados foram lidos integralmente e os dados principais de interesse para esta revisão encontram-se expostos na tabela a seguir (Tabela 1).

Foi encontrada relação da hanseníase com anticorpos anti-fosfolipídeos (aCL e anti- $\beta 2-G P I)$, anti-peptídeo citrulinado cíclico (anti-CCP), fator reumatoide (FR) e anticorpos anti-núcleo (ANA). 
Tabela 1 - Apresentação dos artigos selecionados, de acordo com os autores, ano de publicação, participantes, manifestações clínicas, autoanticorpos e conclusões.

\begin{tabular}{|c|c|c|c|c|c|}
\hline Autor (Ano) & $\begin{array}{l}\text { Tipo de } \\
\text { Estudo }\end{array}$ & Participantes & $\begin{array}{c}\text { Manifestação } \\
\text { Clínica }(n) \\
\end{array}$ & Autoanticorpo (\%) & Conclusão \\
\hline $\begin{array}{l}\text { Ribeiro SLE } \\
\text { et al., } 2011\end{array}$ & Prevalência & $\begin{array}{l}158 \text { pacientes com } \\
\text { hanseníase }\end{array}$ & $\begin{array}{l}\text { TT (6) } \\
\text { BT (32) } \\
\text { BB (25) } \\
\text { BV (35) } \\
\text { VV (56) }\end{array}$ & $\begin{array}{l}\text { anti-b2-GPI (1,4\%) } \\
\text { aCL (4\%) anti-b2-GPI (15,1\%) } \\
\text { aCL (12\%) anti-b2-GPI(15,1\%) } \\
\text { aCL (12\%) anti-b2-GPI }(20,5 \%) \\
\text { aCL }(72 \%) \text { anti-b2-GPI }(46,6 \%)\end{array}$ & $\begin{array}{l}\text { Existe uma alta prevalência de aCL em } \\
\text { pacientes com hanseníase. Houve diferença } \\
\text { significativa para a presença de autoanticorpos } \\
\text { entre pacientes com hanseníase e pacientes } \\
\text { saudáveis }(p<0,01) \text {. }\end{array}$ \\
\hline $\begin{array}{l}\text { Baeza I et } \\
\text { al., } 2012\end{array}$ & Prevalência & $\begin{array}{l}30 \text { pacientes com } \\
\text { hanseníase }\end{array}$ & VV (30) & aCL IgG e IgM (77\%) & $\begin{array}{l}\text { Anticorpos aCL e anti-micolicos estavam } \\
\text { presentes simultaneamente nos pacientes } \\
\text { estudados. }\end{array}$ \\
\hline $\begin{array}{l}\text { Ribeiro SLE } \\
\text { et al., } 2012\end{array}$ & Prevalência & $\begin{array}{l}87 \text { pacientes com } \\
\text { hanseníase }\end{array}$ & $\begin{array}{l}\mathrm{BB}(30) \\
\mathrm{VV}(57)\end{array}$ & ANA positivo $(25,3 \%)$ & $\begin{array}{l}\text { Foi verificada um forte relação entre a } \\
\text { deficiência de vitamina } D \text { e a presença de } \\
\text { autoanticorpos como os anticorpos anti-núcleo. }\end{array}$ \\
\hline $\begin{array}{l}\text { Zavala- } \\
\text { Cerna MG } \\
\text { et al., } 2012\end{array}$ & Prevalência & $\begin{array}{l}67 \text { pacientes com } \\
\text { hanseníase }\end{array}$ & $\begin{array}{l}\text { TT (5) } \\
\text { BB (8) } \\
\text { VV (54) }\end{array}$ & $\begin{array}{c}\text { a-CCP }(60 \%) \text { FR (20\%) } \\
\text { FR }(12,5 \%) \\
\text { a-CCP }(14,8 \%) \text { FR }(12,96 \%)\end{array}$ & $\begin{array}{l}\text { Níveis séricos de anticorpos a-CCP e FR podem } \\
\text { ser utilizados para distinção entre pacientes } \\
\text { tuberculoides e virchowiano. }\end{array}$ \\
\hline $\begin{array}{l}\text { Salvi S e } \\
\text { Chopra A, } \\
2013\end{array}$ & Retrospectivo & $\begin{array}{l}33 \text { pacientes com } \\
\text { hanseníase }\end{array}$ & $\begin{array}{l}\text { TT (9) } \\
\text { VV (22) }\end{array}$ & FR $(26,6 \%)$ ANA positivo $(37,5 \%)$ & $\begin{array}{l}\text { Foi verificada a existencia de erros de } \\
\text { diagnóstico devido o mimetismo de sitomas } \\
\text { entre a hanseníase e doenças autoimunes. }\end{array}$ \\
\hline $\begin{array}{l}\text { Chao G et } \\
\text { al., } 2013\end{array}$ & $\begin{array}{l}\text { Relato de } \\
\text { Caso Clínico }\end{array}$ & $\begin{array}{l}1 \text { paciente, sexo } \\
\text { feminino }\end{array}$ & -- & ANA positivo & $\begin{array}{l}\text { O diagnóstico de hanseníase baseado em } \\
\text { exames laboratoriais pode causar erros de } \\
\text { interpretação. }\end{array}$ \\
\hline $\begin{array}{l}\text { Neder L et } \\
\text { al., } 2014\end{array}$ & Prevalência & $\begin{array}{l}50 \text { pacientes } \\
\text { (crianças e } \\
\text { adolescentes) } \\
\text { com hanseníase }\end{array}$ & -- & $\begin{array}{c}\text { ANA positivo }(2 \%) \quad \text { aCL } \lg M(16 \%) \\
\text { aCL } \operatorname{lgG}(2 \%)\end{array}$ & $\begin{array}{l}\text { Não houve relação entre a presença dos } \\
\text { autoanticorpos e alterações musculares. }\end{array}$ \\
\hline
\end{tabular}

REAS/EJCH | Vol.Sup.38 | e1911 | DOI: https://doi.org/10.25248/reas.e1911.2020 Página 4 de 9 


\section{Revista Eletrônica Acervo Saúde / Electronic Journal Collection Health ISSN 2178-2091}

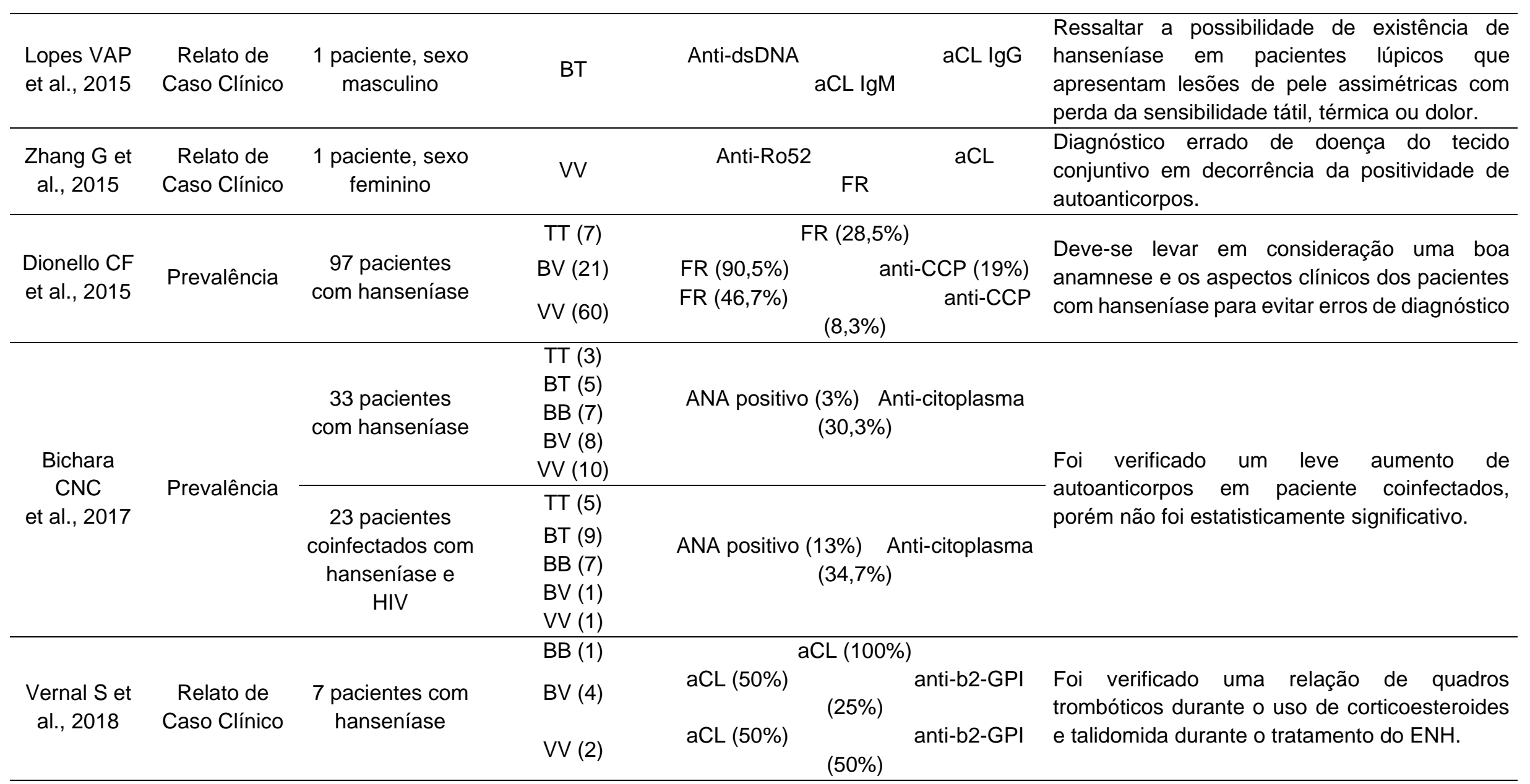

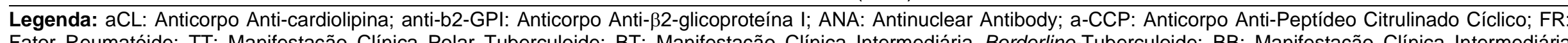
Fator Reumatóide; TT: Manifestação Clínica Polar Tuberculoide; BT: Manifestação Clínica Intermediária Borderline-Tuberculoide; BB: Manifestação Clínica Intermediária Borderline-Bordeline; BV: Manifestação Clínica Intermediária Borderline-Virchowiana; VV: Manifestação Clínica Polar Virchowiana. aCL: Anticorpo Anti-cardiolipina; anti-b2-GPI: Anticorpo Anti-ß2-glicoproteína I; ANA: Antinuclear Antibody; a-CCP: Anticorpo Anti-Peptídeo Citrulinado Cíclico; FR: Fator Reumatóide; DTC: Doença do Tecido Conjuntivo; IgM: Imunoglobulina M; IgG: Iunoglobulina G; HIV: Vírus da Imunodeficiência Humana; ENH: Eritema Nodoso Hansênico; TT: Manifestação Clínica Polar Tuberculoide; BT: Manifestação Clínica Intermediária Borderline-Tuberculoide; BB: Manifestação Clínica Intermediária Borderline-Bordeline; BV: Manifestação Clínica Intermediária BorderlineVirchowiana; VV: Manifestação Clínica Polar Virchowiana. Fonte: Silva GG, 2019.

\footnotetext{
REAS/EJCH | Vol.Sup.38 | e1911 | DOI: https://doi.org/10.25248/reas.e1911.2020 Página 5 de 9
} 


\section{DISCUSSÃO}

Autoanticorpos são proteínas do sistema imunológico que reagem contra elementos próprios, sejam fixos ou solúveis, esta reação irá causar doenças locais ou sistêmicas, como ocorre na Artrite Reumatoide e o Lúpus Eritematoso Sistêmico (LES), respectivamente (SOUZA AWS et al., 2010). Doenças reumatológicas apresentam sinais e sintomas clínicos como lesões de pele, articulações, glânudlas, e ainda, dores e queda de cabelo. Na prática clínica estes sintomas podem indicar facilmente a existência de uma doença autoimune, e, para a confirmação é solicitada a realização de pesquisa de autoanticorpos. $O$ fato de pacientes com hanseníase apresentarem tais sintomas, e além disso, positividade para autoanticorpos, tornou-se um sinal de alerta para reumatologistas com um grande potencial para erros de diagnóstico. Como foi apresentado em todos os artigos de relato de caso clínico selecionados neste revisão, é de extrema importância para o reumatologista a pesquisa por um diagnóstico diferencial entre doenças reumáticas e hanseníase (CHAO G et al., 2013; ZHANG G et al., 2015; LOPES VAP et al., 2015).

No estudo de Ribeiro SLE et al., (2011), os pacientes com a manifestação clínica virchowiana ( $n=56)$ apresentaram maior positividade para autoanticorpos (72\% aCL e 46,6\% anti-b2-GPI) se comparado aos outros grupos com manifestações mais brandas. Em outros estudos, não foi possível diferenciar a positividade de anticorpos entre os grupos de manifestações clínicas, porém, podemos verificar que os polos multibacilares (BB, BV e VV) apresentam maior quantidade de anticorpos anti-fosfolipídeos (APL) e Fator reumatoide (FR), enquanto que nos polos paucibacilares (TT e BT) é possível evidenciar a presença mais significativa de anticorpo anti-peptídeo citrulinado cíclico (anti-CCP). A relevância clínica de cada um destes anticorpos será discutida a frente (ZAVAVA-CERNA MG et al., 2012; DIONELLO CF et al., 2016).

Anticorpos anti-fosfolipídeos (APL) foram citados em seis, dos 12 artigos selecionados para esta revisão (RIBEIRO SLE et al., 2011; BAEZA I et al., 2012; NEDER L et al., 2014; ZHANG G et al., 2015; LOPES VAP et al., 2015; VERNAL $S$ et al., 2018). Os anticorpos APL compõem um grande grupo de anticorpos responsáveis pela fisiopatologia de quadros trombóticos arteriais ou venosos, principalmente durante a gestação, denominada como Síndrome do Anticorpo Antifosfolípide (SAF) (SANTAMARIA JR et al., 2005), fazem parte deste grupo os anticorpos anti-cardiolipina ( $\mathrm{aCL}$ ), anti- $\beta 2$-glicoproteína-I (anti- $\beta 2-\mathrm{GPI}$ ) e anticoagulante lúpico $(\mathrm{AL})$. A presença destes anticorpos em pacientes com hanseníase pode indicar um fator de risco para o desenvolvimento de reações hansênicas ou ainda o fenômeno de lúcio, caracterizado como uma vasculite exacerbada, levando em consideração que estes anticorpos podem estar presentes mesmo sem o aparecimento de manifestações clínicas da SAF (HELMER KA et al., 2004; VERNAL S et al., 2018). O diagnóstico diferencial de SAF e hanseníase sempre deve ser realizado levando em consideração que as duas patologias causam lesões de pele, seja por origem trombótica ou neuropática (SANTAMARIA JR et al., 2005).

O anticorpo anti-peptídeo citrulinado cíclico (anti-CCP) é um importante marcador para a Artrite Reumatóide (AR), apresentando grande especificidade e sensibilidade para o diagnóstico e acompanhamento desta patologia (SILVA AF et al., 2006). Porém, a positividade para este anticorpo na hanseníase pode direcionar o médico a um erro de diagnóstico se o histórico da íntima relação do anti-CCP com a AR for mais relevante que os sintomas e história clínica apresentados pelos pacientes (DIONELLO CF et al., 2016). Nesta revisão, dois trabalhos pesquisaram a relação do anti-CCP com a hanseníase (ZAVALA-CERNA MG et al., 2012; DIONELLO CF et al., 2016).

Anticorpos anti-CCP são de classe IgG, a liberação desses anticorpos é desencadeada mediante apresentação de antígenos proteicos, ou seja, T-dependentes (MESQUITA-JÚNIOR D et al., 2010). Em decorrência deste aspecto, autores sugerem que células $T$ polarizadas na hanseníase apresentam a capacidade de ativar linfócito $B$ espectadores, através do CD40L, e consequentemente induzi-los à produção de autoanticorpos de classe IgG, que são mais específicos (IYER AM et al., 2007; ZAVALA-CERNA MG et al., 2012). Assim como o anti-CCP, o Fator Reumatóide (FR), IgM, é utilizado como um marcador de artrite reumatoide, possuindo menor especificidade se comparado ao anti-CCP. O FR pode estar elevado em doenças infecciosas e outras doenças reumatológicas além da AR (GOELDNER I et al., 2011). 
Nesta revisão, quatro estudos pesquisaram ou relataram a presença de FR na hanseníase (ZAVALACERNA MG et al., 2012; SALVI S e CHOPRA A, 2013; ZHANG G et al., 2015; DIONELLO CF et al., 2016), sendo que no estudo de Dionello CF et al., (2016), 90,5\% dos pacientes hansênicos que apresentavam a manifestação clínica BV apresentaram positividade na pesquisa do FR. O estudo de Zavala-Cerna MG et al., (2012), concluiu que a dosagem de FR e anti-CCP pode ser utilizada para diferenciação dos polos clínicos da hanseníase, TT e VV. As lesões difusas e disseminada na pele presentes na hanseníase VV desencadearam o aparecimento dos anticorpos Anti-Ro52, aCL e FR no caso clínico relatado por Zang G et al., (2015), e Baeza I et al., (2012) verificou positidade de anticorpos aCL IgG e IgM em 23, de 30, pacientes com hanseníase VV.

O teste de triagem para doenças autoimunes utilizado em todo o mundo é a pesquisa de Autoanticorpos em Células HEp-2, também denominado como pesquisa do Fator Anti-núcleo (FAN), do inglês Antinuclear Antibody (ANA). Este teste apresenta grande sensibilidade e baixa especificidade, o que permite a sua utilização para triagem de autoanticorpos. O FAN é um exame de Imunofluorescência Indireta (IFI) e seu resultado é dado em padrões de fluorescência em células HEp-2 (American Type Culture Collection CCL-23), cada padrão é característico da presença de determinado autoanticorpo. A partir do padrão, o médico assistente poderá solicitar a dosagem dos autoanticorpos específicos. Em decorrência das melhorias realizadas nesta técnica, hoje podemos detectar mais de 27 padrões de fluorescência que são distribuídos em nucleares, nucleolares, citoplasmáticos ou mistos, de acordo com as diretrizes do Consenso Brasileiro para Pesquisa de Autoanticorpos em Células HEp-2 - CBA (CRUVINEL WM et al., 2019).

Nesta revisão bibliográfica, sete estudos relataram a presença de ANA em pacientes com hanseníase (RIBEIRO SLE et al., 2012; SALVI S e CHOPRA A, 2013; CHAO G et al., 2013; NEDER L et al., 2014; ZHANG G et al., 2015; LOPES VAP et al., 2015; BICHARA CNC et al., 2017), porém somente dois especificaram os autoanticorpos como anti-dsDNA (LOPES VAP et al., 2015) e anti-Ro52 (ZHANG G et al., 2015).

Anticorpos nucleares são característicos de LES, Síndrome de Sjögren, entre outras doenças autoimunes (CRUVINEL WM et al., 2019), a presença destes anticorpos na hanseníase pode desencadear sintomas característicos de autoimunidade, dificultando o diagnóstico diferencial, pois os pacientes apresentam tanto os sintomas quanto os resultados laboratoriais positivos.

Com base nos estudos apresentados, podemos verificar o potencial imunomodulador do Mycobacterium leprae, tornando esta infecção ainda mais complexa. Em diversos estudos podemos verificar a potencialidade imunomoduladora de alguns agentes infecciosos, como o patógeno $O$. volvulus que ocasiona distúrbios epilépticos na síndrome de Nodding em decorrência de atividade autoimune desencadeada por mimetismo celular (JOHNSON TP et al., 2017), ou ainda como foi dito por um dos artigos pesquisados nesta revisão sobre a associação de anticorpos aCL e anticorpos anti-micólicos, produzidos contra a parede celular do $M$. leprae, na produção de manifestações clínicas reumatológicas (BAEZA I et al., 2012).

\section{CONCLUSÃO}

É inevitável associarmos a presença de autoanticorpos em pacientes hansênicos que apresentam manifestações reumatológicas com a possibilidade de erro de diagnóstico, este tópico foi levantado pela maioria dos trabalhos estudados. Com base no que foi apresentado podemos concluir que durante a infecção pelo Mycobacterium leprae é possível detectar autoanticorpos, sendo que pacientes com manifestações clínicas mais próximas do polo Virchowiano, ou seja, pacientes que apresentam resposta imunológica Th2, são mais propícios ao aparecimento de sintomas reumatológicos e positividade para autoanticorpos. Com base nos resultados também podemos verificar a baixa quantidade de estudos experimentais que tenham objetivo de compreender a imunopatologia e imunomodulação causada pelo Mycobacterium leprae. Além disso, é necessária a realização de estudos que avaliem a relação das manifestações clínicas da hanseníase com os aspectos epidemiológicos e sociais dos pacientes, associando com sintomas autoimunes e positividade para autoanticorpos com propósito de compreender a imunomodulação desencadeada por esta bactéria. 


\section{REFERÊNCIAS}

1. BAEZA I, et al. Lepromatous leprosy patients produce antibodies that recognise non-bilayer lipid arrangements containing mycolic acids. Mem Inst Oswaldo Cruz, 2012; 107: 95-103.

2. BICHARA CNC, et al. Prevalence of autoantibodies against cellular antigens in patients with HIV and leprosy coinfection in the Amazon region Multilingual abstracts. Infectious Diseases of Poverty, 2017; 6: 80.

3. CHAMBERLIN WM, NASER SA. Integrating theories of the etiology of Crohn's disease. On the etiology of Crohn's disease: questioning the hypotheses. Med Sci Monit, 2006; 12(2):27-33.

4. $\mathrm{CHAO}$ G, et al. Leprosy with ANA positive mistaken for connective tissue disease. Clinical Rheumatology, 2013; 32 : 645-648.

5. COSSERMELLI-MESSINA W, COSSERMELLI W. Humoral immunity in Hansen's Disease. São Paulo Medical Journal/RPM, 1995, 113(4):929-934.

6. CRUVINEL WM, et al. V Brazilian consensus guidelines for detection of anti-cell autoantibodies on hep-2 cells. Advances in Rheumatology, 2019; 59:28.

7. CUSICK MF, et al. Molecular Mimicry as a Mechanism of Autoimmune Disease. Clin Rev Allergy Immunol., 2012; 42(1):102-111.

8. DIONELLO CF, et al. Evaluation of rheumatoid factor and anti-citrullinated peptide antibodies in relation to rheumatological manifestations in patients with leprosy from Southern Brazil. International Journal of Rheumatic Diseases, 2016; 19(10):1024-1031.

9. GOELDNER I, et al. Artrite reumatoide: uma visão atual. J Bras Patol Med Lab, 2011; 47(5):495-503.

10. GUPTA SK. Histoid leprosy: review of the literature. International Journal of Dermatology, 2015; 54(11):1283-1288.

11. HANSEN G. On the Etiology of Leprosy. Chirurgical Rewiew, 1875; 55:459-89.

12. HELMER KA, et al. Fenômeno de Lúcio (eritema necrosante) na gestação. An Bras Dermatol, 2004; 79(2): 205210.

13. HERRATH MG, et al. Microorganisms and autoimmunity: making the barren field fertile? Nature Reviews Microbiology, 2003; 1(2):151-157.

14. IYER AM, et al. Leprosy-specific B-cells within cellular infiltrates in active leprosy lesions. Human Pathology, 2007; 38(7):1065-1073.

15. JOHNSON TP, et al. Nodding syndrome may be an autoimmune reaction to the parasitic worm Onchocerca volvulus. Science Translational Medicine, 2017; 9:377.

16. LOPES VAP, et al. Borderline tuberculoid leprosy in childhood onset systemic lupus erythematosus patient. Lupus, $2015 ; 24(13): 1448-1451$.

17. MESQUITA-JÚNIOR D, et al. Sistema Imunitário-Parte II Fundamentos da resposta imunológica mediada por linfócitos T e B. Rev. Bras. Reumatol.,2010; 50(5): 552-580.

18. NAAFS B. World Leprosy Day 2018: How forward respecting the past? Indian Journal of Medical Research, 2018, 147: $1-3$.

19. NEDER L, et al. Musculoskeletal manifestations and autoantibodies in children and adolescents with leprosy. Jornal de Pediatria, 2014; 90(5):457-463.

20. PAWAR M, ZAWAR V. Mid-borderline leprosy masquerading as an overlap syndrome. Rheumatology, 2018; 57(9): 1686-88.

21. PERL A. Pathogenesis and Spectrum of Autoimmunity. Methods Mol Med., 2012; 900:1-9.

22. QUARESMA JAS, et al. HTLV-1, Immune Response and Autoimmunity. Viruses, 2016; 8(5):1-10.

23. RAMAL C, et al. Diffuse Multibacillary Leprosy of Lucio and Latapí with Lucio's Phenomenon, Peru. Emerging Infectious Diseases, 2017; 23(11): 192-193.

24. RIBEIRO SLE, et al. The development of arthritis and antinuclear antibodies correlate with serum 25-hydroxyvitamin D levels in patients with leprosy. Annals of the Rheumatic Diseases, 2012; 71(12): 2062-2063.

25. RIBEIRO SLE, et al. Anti-b2-glycoprotein i antibodies are highly prevalent in a large number of brazilian leprosy patients. Acta Reumatol Port, 2011; 36:30-37.

26. RIDLEY DS, JOPLING WH. A classification of leprosy for research purposes. Leprosy review, 1962; 33:119-28.

27. RIDLEY DS, JOPLING WH. Classification of leprosy according to immunity. A five-group system. International journal of leprosy and other mycobacterial diseases : official organ of the International Leprosy Association, 1966; 34(3): 255-73.

28. SADHU S, MITRA DK. Emerging Concepts of Adaptive immunity in Leprosy. Front. Immunol, $2018 ; 9(9): 604$.

29. SALVI S, CHOPRA A. Leprosy in a rheumatology setting: a challenging mimic to expose. Clin Rheumatol, 2013; 32:1557-1563.

30. SAMARKOS M, VAIOPOULOS G. The Role of Infections in the Pathogenesis of Autoimmune Diseases. Current Drug Target -Inflammation \& Allergy, 2005; 4(1):99-103. 
31. SANTAMARIA JR, et al. Síndrome antifosfolípide. An Bras Dermatol, 2005; 80(3):225-39.

32. SFRISO P, et al. Infections and autoimmunity: the multifaceted relationship. Journal of Leukocyte Biology, 2010; 87(3):385-395.

33. SILVA AF, et al. Valor Diagnóstico do Anticorpo Antipeptídeo Citrulinado Cíclico na Artrite Reumatóide. Rev Bras Reumatol., 2006; 46(3):174-180.

34. SOUZA AWS, et al. Sistema Imunitário - Parte III O delicado equilíbrio do sistema imunológico entre os pólos de tolerância e autoimunidade. Rev Bras Reumatol, 2010; 50(6):665-94.

35. TALHARI C, et al. Clinical aspects of leprosy. Clinics in Dermatology, 2015; 33(1): 26-37.

36. VERNAL S, et al. Anti-phospholipid syndrome in seven leprosy patients with thrombotic events on corticosteroid and/or thalidomide regimen: insights on genetic and laboratory profiles. Rev Soc Bras Med Trop, 2018; 51(1):99_ 104.

37. YANG SH, et al. The molecular basis of immune regulation in autoimmunity. Clinical Science, 2018; 132(1):43-67.

38. ZAVALA-CERNA MG, et al. Anti-cyclic citrullinated peptide antibodies and rheumatoid factor sera titers in leprosy patients from Mexico. Rheumatology International, 2012; 32:3531-3536., 2012.

39. ZHANG G, et al. Cutaneous nodules with positive autoantibodies: histoid leprosy. The Lancet, 2015; 386(10006):1915-1916. 